Methods Web-based questionnaire (Survey monkey ${ }^{\circledR}$ ), developed by the NEonatal Sepsis Trial NETwork (http://www.nest-net.org), was sent to neonatologists worldwide. Questions regarding management $(n=7)$ were introduced by scenarios levelled to low-, medium- and high risk for neonatal EOS. Demographic questions $(n=4)$ are based on competency, caseload, experience of fatal cases (deaths) and country of origin.

Results 439 Neonatologist from 10 countries participated. Laboratory investigations are used in $31 \%$ to start, and in $72 \%$ to stop antibiotic treatment. The decision regarding stop of antibiotic therapy is mainly dependent on conventional laboratory investigations. Only a minority uses newer infection markers as procalcitonin (17\%) or interleukins (9\%). There is a high variance in when to start and when to stop antibiotic therapy with a national distribution. Variance is lower within one country compared to the variance in all participating countries. There is no dependency on other demographic variables.

Conclusions There is a high variance in the management of neonatal EOS. Discontinuation has a high dependency on laboratory infection markers. Clinical research should focus on safety and predictive values of (new) infection markers to support the decision to stop antibiotic therapy early and prevent possibly unnecessary antibiotic treatment.

\section{PO-0254 TUBERCULOSIS OUTBREAK IN A DAYCARE CENTRE}

${ }^{1}$ A Vásquez, ${ }^{1} \mathrm{O}$ Calavia, ${ }^{1} \mathrm{M}$ Alvarez, ${ }^{2} \mathrm{~J}$ Ventosa, 'L Fernandez, ${ }^{1} \mathrm{R}$ Closa. ${ }^{1}$ Pediatrics, Hospital Joan XXIII, Tarragona, Spain; ${ }^{2}$ Pulmonology, Hospital Joan XXIII, Tarragona, Spain

\subsection{6/archdischild-2014-307384.904}

Objetives The control of tuberculosis (TB) transmission and prevention of outbreaks requires appropriate studies for the contacts. We're going to present an outbreak of tuberculosis in a daycare centre.

Methods A case of pulmonary TB were reported in a 3 years old boy from a daycare, admitted with pneumonia without response to conventional antibiotic therapy, tuberculin test (PPD):5 mm, positive quantiferon. Study of family contacts was negative.

After that pulmonary $\mathrm{TB}$ was confirmed in a caregiver from the daycare, she was considered baciliferus and also the index case.

Results Contact study was performed in 90 persons exposed. $85 \%(77 / 90)$ contacts from the daycare (67 children under 3 years and 10 adults). 15 people had positive tuberculin, 8 contacts from the daycare ( 7 children and 1 caregiver). 5 patients of the group mentioned before were considered latent tuberculosis infection, receiving secondary chemoprophylaxis and 3 were diagnosed with tuberculosis disease, being treated with satisfactory outcome in all cases.

The index case had over a month off work so that children with negative tuberculin was repeated at 5 weeks being negative in all cases.

Conclusions TB in children can be confused with other typical lung infections, however, must be ruled out if it has a subacute respiratory symptoms and poor response to conventional treatment. It's really important the rapid detection of contacts incase of adults with TB disease, especially if those work with susceptible people like children. The PPD is still an easy and simple tool for unvaccinated contacts diagnostic.

\section{PO-0255 FEATURES OF HOSPITALISED CHILDREN SUSPECTED INFECTION WITH INFLUENZA A H1N1 SUBTYPE IN PAEDIATRICS OF TEACHING HOSPITAL ALCIDES CARNEIRO (HEAC), PETRÓPOLIS, RJ, BRAZIL}

${ }^{1} \mathrm{E}$ Veiga, ${ }^{2} \mathrm{~A}$ Veiga, ${ }^{3} \mathrm{C}$ Tammela, ${ }^{3} \mathrm{~F}$ Ferreira, ${ }^{3} \mathrm{~J}$ Souza, ${ }^{4} \mathrm{~S}$ Nogueira, ${ }^{4} \mathrm{~S}$ Cordeiro, ${ }^{4} \mathrm{~F}$ Moliterno, ${ }^{4} \mathrm{~N}$ Moliterno. ${ }^{1}$ Pediatria, Faculdade Medicina de Petrópolis, Petrópolis, Brazil; ${ }^{2}$ Pediatria, Faculdade Medicina de Petrópolis, Petrópolis, Brazil; ${ }^{3}$ Graduação Em Medicina, Faculdade Medicina de Petrópolis, Petrópolis, Brazil; ${ }^{4}$ Pediatria, Faculdade Medicina de Petrópolis, Petrópolis, Brazil

\subsection{6/archdischild-2014-307384.905}

Introduction Influenza A virus subtype H1N1 has been identified as an important pathogen in childhood morbidity. Taking into account the post-pandemic situation in 2009, knowledge of the epidemiology of influenza in the paediatric population is essential for better targeting of strategies for clinical management, therapeutic and epidemiological control.

Objective To describe the profile of children hospitalised with suspected H1N1 infection in the paediatric ward of HEAC, during the year 2013.

Methods Cross-sectional, retrospective study, simple analytical paediatric hospitalizations for suspected H1N1.

Results and discussion During the study period 1039 paediatric hospitalizations were reported, including 349 (33\%) for respiratory diseases. Of these, 21 (6\%) used Oseltamivir Phosphate for suspected $\mathrm{H} 1 \mathrm{~N} 1$ from June, focusing more in winter and spring. The age ranged between $2 \mathrm{~m}$ and 11th with an average of 3.16 years. Male predominance $76.19 \%$. The mean hospital stay was 10,33 days. Comorbidities such as encephalopathy, heart diseases, obesity in 17 (80.9\%), and age <2 years in 10 (47.6\%). Were referred to the Intensive Therapy $23.8 \%$. Specific oropharyngeal swabs collected 9 (42.85\%), 3 of these $(14.28 \%)$ confirmed the diagnosis. All were classified as Severe Acute Respiratory Syndrome, as the Protocol of the Ministry of Health Only $6(28.5 \%)$ began specific therapy within the first $48 \mathrm{~h}$, as recommended as an effective treatment.

Conclusion Influenza will remain a challenge for medicine over the years, requiring proactive management. The epidemiological report suspected front, start the appropriate therapy, the specific diagnostic screening and recognition of clinical severity, can contribute significantly in the control of H1N1.

\section{PO-0256 ENTEROVIRUS MENINGITIS: A CASE SERIES REVIEW}

${ }^{1} \mathrm{C}$ Warren, ${ }^{2} \mathrm{P}$ Chakrabarti, ${ }^{1} \mathrm{Y}$ Kumar. ${ }^{1}$ Neonatal Unit, Royal Cornwall Hospital, Truro, UK; ${ }^{2}$ Clinical Microbiology, Royal Cornwall Hospital, Truro, UK

\subsection{6/archdischild-2014-307384.906}

Background and aims Meningitis due to Enterovirus infection is usually mild and self-limiting, particularly affecting young infants. We found that we were increasingly diagnosing the condition and undertook a retrospective review, exploring case characteristics and whether increasing use of virology detection reduced antibiotic course length, having previously rarely employed these techniques.

Methods All cases identified as being diagnosed with meningitis with a positive enterovirus polymerase chain reaction (PCR) result between January 2012 and December 2012, below 16 years of age, were retrospectively reviewed.

Results Nine cases of enterovirus meningitis were identified in the time period. Seven were infants aged 3 months or less, with two patients aged 11 and 15 years respectively. There were five 